\title{
PROPIEDADES PSICOMÉTRICAS DEL INVENTARIO DE ANSIEDAD DE BECK (BAI) EN PACIENTES CON CÁNCER
}

\author{
PSYCHOMETRIC PROPERTIES OF THE BECK ANXIETY INVENTORY (BAI) FOR \\ PATIENTS WITH CANCER
}

Oscar Galindo Vázquez'1,2, Edith Rojas Castillo2, Abelardo Meneses García ${ }^{3}$, José Luis Aguilar Ponce ${ }^{4}$, Miguel Ángel Álvarez Avitia ${ }^{5}$ y Salvador Alvarado Aguilar ${ }^{2}$



Resumen

La sintomatología ansiosa es uno de los principales problemas psicológicos en pacientes oncológicos. La Escala de Ansiedad de Beck (BAI) ha demostrado ser un instrumento válido y confiable. Sin embargo, hasta ahora no se había documentado su comportamiento psicométrico en población oncológica en México.

Objetivo: Determinar las propiedades psicométricas de la Escala de Ansiedad de Beck (BAI) en una muestra de pacientes con cáncer.

Material y Método: participaron 250 pacientes del Instituto Nacional de Cancerología, de los cuales 138 eran mujeres (55,2\%) y 112 eran hombres $(44,8 \%)$; la edad promedio fue de 46,6 $\pm 14,3$ años. Los participantes contestaron además del BAI, la Subescala de ansiedad de la Escala de Ansiedad y Depresión (HADS-A).

Resultados: El análisis factorial varimax con 21 reactivos presentó una estructura con 4 factores: Subjetivo, Neurofisiológico, Autonómico y Síntomas vasomotores que explicaron el $46,38 \%$ de la varianza. La consistencia interna de la escala global mostró un índice satisfactorio $(\alpha=0,82)$. La validez por medio de correlación con el HADS-A mostraron resultados significativos ( $r$ de Pearson de $0,58, p<0,05$ ) y el ECOG ( $r$ de Pearson de $-0,36 p<0,05$ ).
Abstract

Introduction: Depressive symptomatology is a major mental health problem in cancer patients. The Beck Anxiety Scale (BAI) has proven to be a reliable and valid instrument. However, until now has not been documented it's psychometric properties in Mexican population with cancer.

Objetives: Determine the psychometric properties of the Beck Anxiety Scale BAI in a sample of cancer patients.

Material and methods: Involved 250 patients from the National Cancer Institute, of which 112 were women $(55.2 \%)$ were male and $138(44.82 \%)$; the average age was $46.6 \pm$ 14.3 years. Participants answered the BAI, and also the Anxiety Sub-scale of HADS.

Results: A varimax factor analysis with 21 items show a structure of 4 factors: Subjective, Neurophysiological, Autonomic and vasomotor symptoms that explain $46.38 \%$ of the variance. The internal consistency of the overall scale was satisfactory $(\alpha=0.82)$. The validity through correlations with the HADS-A showed significant associations (Pearson $r=0.58, p$ $<0.05$ ) and ECOG (Pearson -0.36, p <0.05).

Conclusions: The BAI in Mexican population with cancer showed adequate psychometric characteristics. Detection of anxiety symp-

\section{Correspondencia:}


Conclusiones: el BAI en pacientes con cáncer en población mexicana presentó adecuadas características psicométricas. La detección de sintomatología ansiosa a través del BAI deriva en beneficios para la población oncológica $y$ en estrategias funcionales de atención adecuada y costo-efectivas.

Palabras clave: Ansiedad, Cáncer, Pacientes, México, Propiedades psicométricas. toms through BAI provides benefits to the cancer population and facilitates the development of adequate and cost-effective care strategies.

Keywords: Anxiety, Cancer, Patients, Mexico, Psychometric properties.

\section{INTRODUCCIÓN}

Durante el proceso oncológico, la ansiedad tiende a reflejar una reacción ante el diagnóstico y el tratamiento ${ }^{(1)}$.

Se considera un estado emocional en el que el individuo se siente tenso, nervioso y preocupado asociado con sintomatología física como palpitaciones, temblor o sudoración $^{(2,3)}$.

La prevalencia de ansiedad en población oncológica es de 0,9\% a 49\% con instrumentos de autoreporte ${ }^{(4,5)}$. Sin embargo estudios que utilizan entrevistas psiquiátricas estandarizadas y criterios diagnósticos identifican un rango más estrecho de $10 \%$ a $30 \%{ }^{(3)}$.

Se considera que la ansiedad es adaptativa si es proporcional a la amenaza, es transitoria, solo dura mientras persiste el estímulo temido y si facilita la puesta en marcha de recursos. Sin embargo, se considera desadaptativa cuando es desproporcionada a la amenaza, implica un aumento anómalo de la frecuencia, intensidad o duración de los síntomas y se mantiene en el tiempo ${ }^{(6,7)}$.

La presencia de ansiedad ha sido documentada a lo largo del tratamiento oncológico, en el diagnóstico ${ }^{(8-10)}$, recidiva, cuando el paciente está esperando el tratamiento ${ }^{(1,11)}$ así como en estadios avanzados ${ }^{(10)}$.

La ansiedad puede interferir con la capacidad de afrontamiento al tratamiento, afectar la calidad de vida ${ }^{(2,12,13)}$, disminuir la capacidad para tolerar los efectos secundarios del mismo ${ }^{(2)}$, afectar el funcionamiento social y contribuir a la intensificación de anorexia, fatiga, náuseas, vómito y mayor deterioro físico general ${ }^{(12,14)}$.

Esto puede derivar en una falta de reconocimiento de sintomatología ansiosa por parte del equipo médico, atribuyéndola a una respuesta normal e incertidumbre asociada al curso y tratamiento oncológico ${ }^{(13)}$.

Por lo que es fundamental evaluar la ansiedad en población oncológica. Los instrumentos de evaluación de ansiedad pueden clasificarse en dos grupos: a) Instrumentos que evalúan sintomatología ansiosa y b) Escalas para diagnóstico de trastornos de ansiedad ${ }^{(15)}$.

El Inventario de Ansiedad de Beck (BAI) es uno de los instrumentos más empleados en población clínica y no clínica para evaluar sintomatología ansiosa, se ha estudiado tanto en población general como psiquiátrica, adolescentes y adultos, mostrando una alta consistencia interna (alfa superior a 0,90), así como una validez convergente mayor a 0,50 (15-19).

El BAI se administra habitualmente antes y después del tratamiento, indicando una reducción estadísticamente significativa en la puntuación media como una respuesta positiva al tratamiento ${ }^{(20)}$.

El BAI puede ayudar a responder a la pregunta de si un paciente está igual, ha disminuido o incrementado su ansiedad de forma clínicamente significativa ${ }^{(21)}$. 
Sin embargo no se tienen datos sobre su validez en población mexicana con cáncer. Por lo que el objetivo de este estudio fue obtener sus propiedades psicométricas en una muestra de pacientes con cáncer.

\section{MÉTODO}

\section{Participantes}

Se incluyeron 250 pacientes mexicanos con cáncer de ambos sexos entre 16 y 78 años (ver la tabla 1 para una descripción de la muestra). La muestra fue obtenida por disponibilidad durante el periodo comprendido de Enero a Junio de 2014 en el Instituto Nacional de Cancerología de México (INCan).

\section{Procedimiento}

El personal del servicio de Psico-oncología realizó la aplicación de los instrumentos en consulta externa y hospitalización. El proyecto fue aprobado por los Comités Científico y de Ética en Investigación del INCan. Cada uno de los participantes comprendió y firmo el consentimiento informado de investigación.

Los criterios de inclusión fueron: 1). En tratamiento oncológico (cualquier tipo de tratamiento), 2) Cualquier tipo de diagnóstico oncológico y estadio, 3) Saber leer y escribir. Criterios de exclusión: 1) Disnea severa, 2) Deficiencias cognitivas, 3) Problemas auditivos y 4) Afectaciones visuales severas.

\section{Tabla 1. Descripción de la muestra}

\begin{tabular}{|c|c|c|c|c|c|}
\hline & $f$ & $\%$ & & f & $\%$ \\
\hline $\mathbf{N}$ & 250 & 100 & Diagnóstico & & \\
\hline \multirow[t]{2}{*}{ Edad (Rango) 18-77 } & & & Mama & 98 & 38,7 \\
\hline & & & Ginecológicos & 57 & 23,3 \\
\hline Sexo & & & Pulmón & 36 & 15,1 \\
\hline Masculino & 112 & 44,8 & Cabeza y cuello & 28 & 11,5 \\
\hline \multirow[t]{2}{*}{ Femenino } & 138 & 55,2 & Gástricos & 20 & 7,8 \\
\hline & & & Otros & 11 & 3,6 \\
\hline \multicolumn{6}{|l|}{ Escolaridad } \\
\hline Ninguno & 14 & 5,6 & Estadio & & \\
\hline Primaria & 61 & 24,4 & I & 33 & 13,8 \\
\hline Secundaria & 74 & 29,6 & II & 52 & 20,8 \\
\hline Bachillerato & 59 & 23,6 & III & 93 & 37,2 \\
\hline \multirow[t]{2}{*}{ Licenciatura } & 42 & 16,8 & IV & 72 & 28,2 \\
\hline & & & Tratamiento & & \\
\hline Estado civil & & & QT & 104 & 41,6 \\
\hline Soltero(a) & 62 & 24,8 & $\mathrm{QT}+\mathrm{RT}$ & 64 & 25,6 \\
\hline Casado o Unión libre & 148 & 59,2 & Cirugía & 60 & 24,0 \\
\hline Divorciado(a) & 26 & 10,4 & Radioterapia & 22 & 8,8 \\
\hline \multirow[t]{2}{*}{ Viudo } & 14 & 5,6 & & & \\
\hline & & & ECOG & & \\
\hline Paternidad & & & 0 & 107 & 42,8 \\
\hline $\mathrm{Si}$ & 198 & 79,2 & 1 & 109 & 43,6 \\
\hline \multirow[t]{2}{*}{ No } & 52 & 20,8 & 2 & 23 & 9,1 \\
\hline & & & 3 & 11 & 4,5 \\
\hline
\end{tabular}

Nota: QT= Quimioterapia, RT=Radioterapia. 


\section{Instrumentos}

Inventario de Ansiedad de Beck (BAI)

El BAI (Beck, Epstein, Brown y Steer, 1988)(16) estandarizado para población mexicana por Robles, Varela, Jurado y Páez (2001)(15), es un instrumento de auto-reporte que consta de 21 reactivos que determinan la severidad de las categorías sintomáticas y conductuales. El BAI ha demostrado una alta consistencia interna (alfas superiores a 0,90), validez divergente moderada (correlaciones menores a 0,60$)$, y validez convergente adecuada (correlaciones mayores a 0,50$)^{(15)}$. Las propiedades psicométricas del $\mathrm{BAI}$ en población mexicana presentan una alta consistencia interna (alfas de Chorach de 0,84 y 0,83$)$, un alto coeficiente de confiabilidad test-retest $(r=0,75)$, validez convergente adecuada (índices de correlación entre el BAI y el Inventario de Ansiedad Rasgo Estado -IDARE- moderados y positivos) y una estructura factorial de cuatro factores principales denominados: a) subjetivo, b) neurofisiológico, c) autonómico y d) pánico(15).

Subescala de ansiedad de la Escala Hospitalaria de Ansiedad y Depresión (HADS-A). El HADS-A Zigmond y Snaith $(1983)^{(22)}$ es una subescala autoaplicada conformada por 7 ítems que puede utilizarse en medios hospitalarios no psiquiátricos o en atención primaria. Considera dimensiones cognitivas y afectivas, excluyendo la dimensión somática. En México el HADS-A ha sido validado por Galindo et al. $(2015)^{(23)}$ en pacientes con cáncer, con una consistencia interna satisfactoria $(\alpha=0,86)$ y una validez concurrente de 0,514 a 0,849 .

\section{Escala ECOG}

Diseñada por el Eastern Cooperative Oncology Group (ECOG) ${ }^{(24)}$. Su principal función es determinar el status de funcionalidad y capacidades del paciente en su vida diaria. La escala ECOG se puntúa de 0 a 5, sus valores son: 0 . Paciente totalmente asintomático y capaz de realizar trabajos y actividades normales. 1. Presenta síntomas que le impiden realizar trabajos arduos, aunque se desempeña normalmente en sus actividades diarias. 2. No es capaz de desempeñar ningún trabajo, se encuentra con síntomas que le obligan a permanecer en cama durante varias horas al día, no superan el $50 \%$ de este. Satisface la mayoría de sus necesidades personales solo. 3 . Esta en cama más de la mitad del día por la presencia de síntomas. Necesita ayuda para la mayoría de las actividades diarias. 4. Permanece en cama el $100 \%$ del día y necesita ayuda para todas las actividades de la vida diaria. 5. Paciente fallecido.

\section{Análisis estadísticos}

Se efectuó estadística descriptiva para caracterizar a la muestra con base en los datos sociodemográficos y clínicos.

Se determinó la estructura factorial por medio de un análisis de componentes principales con rotación Varimax. Los criterios utilizados para el análisis factorial fueron: 1) cargas factoriales $\geq 0,40$; 2) factores con por lo menos tres ítems, y 3) coeficiente de consistencia interna por cada factor alfa de Cronbach $\geq 0,60$. La relación entre el $\mathrm{BAI}$, el HADS-A y el ECOG se analizó mediante el coeficiente de correlación de Pearson. El valor alfa establecido fue de 0,05.

\section{RESULTADOS}

Las puntuaciones medias en el BAI para hombres fue $12,12(+7,40)$ y para mujeres $11,41(+7,22)$; el puntaje total promedio de la muestra fue de $12,68(+7,53)$. No se encontraron diferencias estadísticamente significativas. 


\section{Estructura factorial}

El análisis factorial obtuvo en la prueba de esfericidad de Bartlett una $(p=0,001)$ y un valor de 0,83 en la prueba de KaiserMeyer-Olkin.

El análisis factorial con rotación varimax identificó un modelo con cuatro factores con autovalores arriba de 1,00 que en conjunto explican el $46,38 \%$ de la varianza; los cuales resultaron similares a la versión original.

\section{Consistencia interna}

El análisis de consistencia interna del BAI demostró un alfa de Cronbach de los
21 reactivos de 0,82 , así como alfas de 0,78 a 0,62 en los 4 factores. La consistencia puede considerarse adecuada (ver la tabla 2).

\section{Validez externa}

La tabla 3 muestra las asociaciones entre el BAI total, sus sub-escalas y el HADSA. Las correlaciones fueron positivas y significativas ( $r$ de Pearson de 0,85 a 0,19, $p<0,05)$. La correlación entre el BAl y el índice ECOG mostró correlaciones inversamente proporcionales ( $r$ de Pearson de $-0,36$ a $-0,22, p<0,05)$.

\section{Tabla 2. Análisis factorial de elementos del BAI}

\begin{tabular}{|c|c|c|c|}
\hline $\begin{array}{l}\text { Escala Global } \alpha=0,82 \\
\text { Varianza Explicada }=46,37 \%\end{array}$ & Carga factorial & $\begin{array}{l}\text { Varianza } \\
\text { explicada }\end{array}$ & $\begin{array}{c}\text { Alfa de } \\
\text { Cronbach }\end{array}$ \\
\hline Factor 1. Subjetivo & & $25,74 \%$ & $\alpha=0,78$ \\
\hline 4. Incapacidad de relajarme. & 0,421 & & \\
\hline 5. Miedo a que suceda lo peor. & 0,782 & & \\
\hline 8. Sensación de inestabilidad e inseguridad física. & 0,419 & & \\
\hline 9. Terrores. & 0,630 & & \\
\hline 10. Nerviosismo. & 0,607 & & \\
\hline 14. Miedo a perder el control. & 0,540 & & \\
\hline 16. Miedo a morirme. & 0,784 & & \\
\hline Factor 2. Neurofisiológico & & $7,46 \%$ & $\alpha=0,72$ \\
\hline 1. Hormigueo o entumecimiento. & 0,658 & & \\
\hline 3. Temblor de piernas. & 0,736 & & \\
\hline 6. Mareo o aturdimiento. & 0,658 & & \\
\hline 12. Temblores de manos. & 0,728 & & \\
\hline 13. Temblor generalizado o estremecimiento. & 0,728 & & \\
\hline 17. Sobresaltos. & 0,728 & & \\
\hline 18. Molestias digestivas o abdominales. & 0,728 & & \\
\hline 19. Palidez. & 0,728 & & \\
\hline Factor 3. Autonómico & & $6,73 \%$ & $\alpha=0,64$ \\
\hline 7. Palpitaciones o taquicardia. & 0,753 & & \\
\hline 11. Sensación de ahogo. & 0,628 & & \\
\hline 15. Dificultad para respirar. & 0,736 & & \\
\hline Factor 4. Síntomas vasomotores & & $6,44 \%$ & $\alpha=0,62$ \\
\hline 2. Sensación de calor. & 0,641 & & \\
\hline 20. Rubor facial. & 0,672 & & \\
\hline 21. Sudoración. & 0,614 & & \\
\hline
\end{tabular}


Tabla 3. Correlaciones entre el BAI, HADS-A y ECOG.

\begin{tabular}{lcccccc}
\hline & 1 & 2 & 3 & 4 & 5 & 6 \\
\cline { 2 - 6 } 1. BAI total & & & & & \\
2. Factor 1 & $0,855^{*}$ & & & & \\
3. Factor 2 & $0,852^{*}$ & $0,594^{*}$ & & & & \\
4. Factor 3 & $0,606^{*}$ & $0,381^{*}$ & $0,400^{*}$ & & & \\
5. Factor 4 & $0,513^{*}$ & $0,271^{*}$ & $0,300^{*}$ & $0,196^{*}$ & & \\
6. HADS-A & $0,582^{*}$ & $0,587^{*}$ & $0,460^{*}$ & $0,327^{*}$ & $0,204^{*}$ & \\
7. ECOG & $-0,365^{*}$ & $-0,321^{*}$ & $-0,288^{*}$ & $-0,314^{*}$ & $-0,223^{*}$ & $-0,251^{*}$ \\
\hline
\end{tabular}

*Correlaciones Pearson con $p<0,05$.

\section{DISCUSIÓN Y CONCLUSIONES}

Los análisis psicométricos realizados en esta investigación indican que el BAI Beck, Steer et al. (1996) ${ }^{(16)}$ mantiene una estructura similar a los resultados informados en la literatura ${ }^{(15,16,20,21,25,26)}$. Por su parte autores como Sanz et al. ${ }^{(15)}$ consideran que aunque una solución factorial única es adecuada para el BAI, el hecho es que en cualquiera de los dos planteamientos se recomienda utilizarlo como una sola escala, y no como subescalas individuales.

En relación a los factores identificados, es recomendable prestar atención al factor subjetivo ya que es de utilidad clínica en la identificación del componente afectivo de la ansiedad en un grupo de pacientes en los que la carga sintomática es altamente prevalente ${ }^{(27)}$. En cuanto a los factores Neurofisiológico, Autonómico y Síntomas vasomotores identificados pueden indicar sintomatología frecuente en población oncológica $^{(28,29)}$. Pues de acuerdo a Cleaned $(2007)^{(30)}$ la prevalencia de síntomas como fatiga, dolor, debilidad, tristeza, ansiedad, disnea, vómito y deterioro cognitivo han sido consistentemente reportados en pacientes oncológicos, con un promedio de 10 síntomas durante el periodo de tratamiento y cuatro durante los primeros meses posteriores a este. Adicionalmente Wang et al. (2010) ${ }^{(31)}$ plantea que estos síntomas son el resultado del cáncer y su tratamiento, los cuales incluso pueden persistir años después de terminado el mismo.

En cuanto a la validez convergente del BAI presentó correlaciones moderadas y altas con el HADS-A, y una asociación inversamente proporcional baja con el Índice ECOG que es un índice funcionalidad en población oncológica; lo que respalda la validez convergente del BAI con mediciones de ansiedad que no consideran componentes somáticos e índicadores de funcionalidad.

Como un instrumento de tamizaje, el BAI puede funcionar mejor en los trastornos de ansiedad con un alto componente fisiológico, como el trastorno de pánico, en comparación con los trastornos con un componente central cognitivo, como la fobia social o el trastorno obsesivo-compulsivo $^{(20)}$.

Dado que el BAI contiene solo 21 ítems que se pueden responder en 5-10 minutos, es posible aplicarlo durante el proceso terapéutico para monitorizar los progresos del paciente ${ }^{(21)}$. Resulta adecuado para una población donde la aplicación de instrumentos de screening extensos de manera rutinaria es poco recomendable.

El reconocimiento de niveles patológicos de ansiedad es un aspecto crítico de la atención integral al paciente oncológico ${ }^{(32)}$. Pues la detección del malestar emocional tiene el potencial de influir en la comunicación, el comportamiento clínico y la deri- 
vación de los pacientes ${ }^{(33)}$ a los servicios de psicooncología que repercutirá en un mejor afrontamiento del cáncer y su tratamiento.

\section{Limitaciones y sugerencias}

Se requerirá estudios prospectivos que corroboren la estructura factorial identificada en esta investigación, e identifiquen el comportamiento del BAI a lo largo del tiempo en población oncológica.

\section{REFERENCIAS BIBLIOGRÁFICAS}

1. Linden W, Vodermaier A, MacKenzie R. Anxiety and depression after cancer diagnosis: Prevalence rates by cancer type, gender, and age. J Affect Disor 2012;141:34351. Doi: 10.1016/j.jad.2012.03.025

2. Maté J, Hollenstein M F, Gil FL. Insomnio, ansiedad y depresión en el paciente oncológico. Psicooncología 2004;1:211-30.

3. Stark D, Kiely M, Smith A, Velikova G, House A, Selby P. Anxiety disorders in cancer patients: Their nature, associations, and relation to quality of life. J Clin Oncol 2002;20:3137-48. Doi: 10.1200/ JCO.2002.08.549

4. van't Spijker A, Trijsburg RW, Duivenvoorden HJ. Psychological sequelae of cancer diagnosis: A meta-analytical review of 58 studies after 1980. Psychosom Med 1997;59:280-93. Doi: 10.1097/00006842199705000-00011

5. Ibbotson T, Maguire P, Selby P, Priestman T, Wallace L. Screening for anxiety and depression in cancer patients: The effects of disease and treatment. Eur J Cancer 1994;30:3740. Doi: 10.1016/S0959-8049(05)80015-2

6. Arranz P, Barbero JJ, Barreto P, Bayés R. Protocolos de intervención. En: Arranz P, Barbero JJ, Barreto P, Bayés R. editores. Intervención emocional en cuidados paliativos. Modelos y protocolos. Barcelona: Ariel Ciencias Médicas, 2003; p.51-140.

7. Consedine NS, Adjei B. A, Ramirez PM, McKiernan JM. An object lesson: Source determines the relations that trait anxiety, prostate cancer worry, and screening fear hold with prostate screening frequency. Cancer Epidemiol Biomarkers Prev 2008;17: 1631-9. Doi: 10.1158/10559965.EPI-07-2538

8. Breitbart W. Psycho-oncology: Depression, anxiety, delirium. Semin Oncol 1994;21:754-69.

9. Ciaramella A, Poli P. Assessment of depression among cancer patients: The role of pain, cancer type and treatment. Psychooncology 2001;10:156-65. Doi: 10.1002/pon.505

10. Brown LF, Kroenke K, Theobald DE, Wu J, Tu W. The association of depression and anxiety with health-related quality of life in cancer patients with depression and/or pain. Psychooncology 2010;19:734-41. Doi:10.1002/pon.1627

11. Stark DP, House A. Anxiety in cancer patients. Br J Cancer 2000;83:1261-7.Doi: 10.1054/bjoc.2000.1405

12. Aass N, Fossa SD, Dahl AA, Moe TJ. Prevalence of anxiety and depression in cancer patients seen at the Norwegian Radium Hospital. Eur J Cancer 1997;33:1597-604. Doi:10.1016/S0959-8049(97)00054-3

13. Yu LS, Chojniak R, Borba MA, Girão DS, Lourenço MTDPC. Prevalence of anxiety in patients awaiting diagnostic procedures in an oncology center in Brazil. Psychooncology 2011;20:1242-5. Doi: 10.1002/pon.1842

14. Forester B, Kornfeld D S, Fleiss JL, Thompson S. Group psychotherapy during radiotherapy: Effects on emotional and physical distress. Am J Psychiatry 1993;150:1700-6.

15. Robles R, Varela R, Jurado S, Páez F. Versión mexicana del Inventario de Ansiedad de Beck: propiedades psicométricas. Rev Mex Psicol 2001;18:211-8.

16. Beck AT, Epstein N, Brown G, Steer RA. An inventory for measuring clinical anxiety: psychometric properties. J Cons Clin Psychol 1988;56:893-7. Doi: 10.1037/0022006X.56.6.893 
17. Beck AT, Steer RA. Relationship between the Beck anxiety inventory and the Hamilton anxiety rating scale with anxious outpatients. J Anxiety Disord 1991;5:213-3. Doi: 10.1016/0887-6185(91)90002-B

18. Borden JW, Peterson D. R, Jackson EA. The Beck Anxiety Inventory in nonclinical samples: Initial psychometric properties. J Psychopatholo Behav Assess 1991;13:3456. Doi: 10.1007/BF00960446

19. Steer RA, Rissmiller DJ, Ranieri WF, Beck AT. Structure of the computer-assisted Beck Anxiety Inventory with psychiatric inpatients. J Pers Assess 1993;60:532-42. Doi:10.1207/s15327752jpa6003_10

20. Magán I, Sanz J, García-Vera MP. Psychometric properties of a Spanish version of the Beck Anxiety Inventory (BAI) in general population. Spanish J Psychol 2008;11:626-40. Doi:10.1017/ S1138741600004637

21. Sanz J. Recomendaciones para la utilización de la adaptación española del Inventario de Ansiedad de Beck (BAI) en la práctica clínica. Clín Salud 2014; 25:39-48. Doi: 10.1016/S1130 5274(14)70025-8

22. Zigmond AS, Snaith RP. The Hospital Anxiety and Depression Scale. Acta Psychiatric Scand 1983;67:361-70. Doi: 10.1111/ j.1600-0447.1983.tb09716.x

23. Galindo $O$, Benjet $C$, Juárez $F$, Rojas $E$, Riveros A, Aguilar-Ponce JL, Álvarez MA, Alvarado S. Propiedades psicométricas de la Escala Hospitalaria de Ansiedad y Depresión (HADS) para pacientes con cáncer en población mexicana. Salud Mental 2015; 4(38).

24. Oken MM, Creech RH, Tormey, DC, Horton J, Davis TE, McFadden ET, Carbone PP. Toxicity and response criteria of the Eastern Cooperative Oncology Group Am J Clin Oncol 1982;5:649-56.

25. Lovibond PF, Lovibond SH. The structure of negative emotional states: Comparison of the Depression Anxiety Stress Scales (DASS) with the Beck Depression and Anxiety Inventories. Behaviour re- search and therapy 1995;33:335-43. Doi: 10.1016/0005-7967(94)00075-U

26. Fydrich T, Dowdall D, Chambless DL. Reliability and validity of the Beck Anxiety Inventory. J Anxiety Disord 1992;6:55-61. Doi: 10.1016/0887-6185(92)90026-4

27. Teunissen S, Wesker W, Kruitwagen C, de Haes H, Voest EE, de Graeff A. Review article symptom prevalence in patients with incurable cancer: A Systematic Review J Pain Symptom Manage 2007;34:94-104. Doi: 10.1016/j.jpainsymman.2006.10.015

28. Walker EM, Rodriguez A I, Kohn B, Ball RM, Pegg J, Pocock JR et al. Acupuncture versus venlafaxine for the management of vasomotor symptoms in patients with hormone receptor-positive breast cancer: A randomized controlled trial. J Clin Oncol 2010;28:634-40. Doi: 10.1200/ JCO.2009.23.5150

29. Hammar M, Frisk J, Grimas O, Hook M, Spet AC, Wyon Y. Acupuncture treatment of vasomotor symptoms in men with prostatic carcinoma: A pilot study. J Urol 1999;161:8536. Doi: 10.1016/S0022-5347(01)61789-0

30. Cleeland CS. Symptom Burden: Multiple symptoms and their impact as patientreported outcomes. J Natl Cancer Inst Monogr 2007;37:16-21. Doi: 10.1093/ jncimonographs/lgm005

31. Wang XS, Cleeland CS, Mendoza TR, Yun YH, Wang Y, Okuyama T, Johnson VE. Impact of cultural and linguistic factors on symptom reporting by patients with cancer. J Natl Cancer Inst 2010;102:732-738. Doi: 10.1093/jnci/djq097

32. Rico JL, Restrepo M, Molina M. Adaptación y validación de la escala hospitalaria de ansiedad y depresión (HAD) en una muestra de pacientes con cáncer del Instituto Nacional de Cancerología de Colombia. Avances en Medición 2005;3:73-86.

33. Mitchell AJ, Waller A, Carlson LE. Implementing a screening programme for distress in cancer settings: Science and practice. Psicooncología 2012;9:259-275. Doi. 10.5209/rev_PSIC.2013.v9.n2-3.40896 